\title{
Circular economy and engineering concepts for technology and policy development
}

\author{
Petar Sabev Varbanov ${ }^{1} \cdot$ Timothy Gordon Walmsley $^{1}$
}

Published online: 8 March 2019

๑) Springer-Verlag GmbH Germany, part of Springer Nature 2019

A previous editorial of Clean Technologies and Environmental Policy looked at the fundamental structure of energy supply and use in modern economies, stressing the high share of wasted energy. Based on that, the need has been revealed for more intensive research and development efforts, for minimising the rate of intake of energy and water resources, commonly known as energy and water efficiency.

In that context, the Circular Economy concept emerged in the 1970s, spreading into the domains of Economy and Business Process Management. The focus of the approach is on the minimisation of the intake of fresh raw materials by the means of various reuse and recycling schemes. A good example of implementing this approach is the waste management hierarchy, which initially was summarised as "reuse-recycling-energy recovery-disposal" and has recently developed into the more complete set of priorities: "prevention-minimisation-reuse-recycling-energy recovery-disposal".

Coming from the business management domain, the Circular Economy concept has helped motivate progress on reducing to some extent raw material intake. However, such thinking naturally reaches its limits once all the socalled low-hanging fruits with simple economic drivers are exhausted. Beyond this point, conceptual sketches of an ideal system struggle to provide a sufficiently detailed blueprint to drive greater innovation and step-improvements in industrial and societal efficiency in terms of reductions in resource intake and environmental impacts. The main issue is that this concept emphasises organisation patterns of businesses, while the technological side of the problems is deemed subsidiary, neglecting the importance of engineering considerations and design. This causes some cases

Petar Sabev Varbanov

varbanov@fme.vutbr.cz

1 Sustainable Process Integration Laboratory - SPIL, NETME Centre, Faculty of Mechanical Engineering, Brno University of Technology - VUT, Brno, Czech Republic of resource cascading to be branded as "circular" or "recycling". A good example of this is the manufacture of products with plastic components, where part of the material is recycled from discarded plastic products but not all.

Coming from industrial engineering research, the Process Integration family of methodologies has been very successful at achieving in practice significant reductions in energy and water resource demands, simultaneously with reducing the related environmental impacts of emissions and effluents. The principle, on which Process Integration is built, is to integrate individual process components into highly efficient networks for recovering and reusing the concerned resources and energy. On the example of Heat Integration, Heat Exchanger Networks are used to achieve heat recovery by reusing part of the process heat, simultaneously replacing the use of fuel and external utility cooling. This achieves synergy for the benefit of the whole system. Thermodynamic laws constrain the degree of Heat Integration that is theoretically possible and realistic in practice, leading to the unavoidable demand for external high-quality utility.

While Process Integration applications could prove successful in reducing resource use in support of a Circular Economy, the main success stories to date are from energy and water savings and the mitigation of their environmental impacts. This can be attributed to the engineering focus and thermodynamic origin of the Process Integration methods, where the business and organisational perspectives are not widely represented.

Process Integration offers one tool which has a potential for further conceptual and methodological developments that could enable higher resource efficiency and emission reduction. This tool is the concept of Performance Targets. Targets are identified prior to system design and optimisation, based on thermodynamic principles and model simplification. They typically represent minimum resource demands or minimum emissions possible that cannot be exceeded by the system. This implements in practice the optimisation concept of bounds. Performance Targets, combined with 
an understanding of inherent Pinch Points (or bottlenecks), provide a window into the fundamental limits of the system as well as the essential elements needed for its highly efficient design.

The tool of Performance Targeting can be used beyond the design of resource recovery and reuse networks-including at an economy scale. An inspiration for this can be gathered from the "Process Modifications" reasoning in Heat Integration, where the heat recovery targets are employed to guide a redesign - or a "modification" of the underlying process (e.g. a distillation system), so that to minimise the inherent energy demands, making the problem of heat recovery much easier and cheaper to solve.

From the above discussion, a couple of key lessons can be extracted. The first is related to the observation that both concepts of Circular Economy and Process Integration have the same goals, but have been applied separately, which limits the potential for the practical applications and efficiency increases. The lesson here is that concepts, methods and projects, transferring the knowledge between these two core areas, and exploiting their strengths in synergy, are expected to unlock further potential for improved system performance, based on both the business and engineering perspectives.

Another implication, providing a lesson, is that the considerations relating to both Circular Economy and Process Integration have mainly included resource reuse around core production processes, while the designs of the core processes are usually not tuned to inherently minimise the need for resources. Therefore, further research exploring the inherent resource and pollution minimisation of the production processes is very much necessary, e.g. implementing the "prevention" priority in the waste management hierarchy. This is an area where the use of the Performance Targeting for Process Modifications-one of the Process Integration key tools-may become indispensable. 